\title{
Landscape of somatic mutations in breast cancer: new opportunities for targeted therapies in Saudi Arabian patients
}

\author{
Duna H. Barakeh¹, Rasha Aljelaify², Yara Bashawri³, Amal Almutairi², Fatimah \\ Alqubaishi' ${ }^{2}$ Mohammed Alnamnakani ${ }^{4}$, Latifa Almubarak ${ }^{2}$, Abdulrahman Al \\ Naeem $^{5}$, Fatema Almushawah ${ }^{6}$, May Alrashed ${ }^{7,8}$ and Malak Abedalthagafi ${ }^{2}$ \\ ${ }^{1}$ Department of Pathology, King Saud University Medical City, Riyadh, Kingdom of Saudi Arabia \\ ${ }^{2}$ Genomics Research Department, Saudi Human Genome Project, King Fahad Medical City and King Abdulaziz City for \\ Science and Technology, Riyadh, Kingdom of Saudi Arabia \\ ${ }^{3}$ Department of Biostatistics, Research Centre, King Fahad Medical City, Riyadh, Kingdom of Saudi Arabia \\ ${ }^{4}$ Department of Pathology, King Fahad Medical City, Riyadh, Kingdom of Saudi Arabia \\ ${ }^{5}$ Department of Radiology, King Fahad Medical City, Riyadh, Kingdom of Saudi Arabia \\ ${ }^{6}$ Department of Surgery, King Fahad Medical City, Riyadh, Kingdom of Saudi Arabia \\ ${ }^{7}$ Department of Clinical Laboratory Sciences, College of Applied Medical Sciences, King Saud University, Riyadh, Kingdom of \\ Saudi Arabia \\ ${ }^{8}$ Chair of Medical and Molecular Genetics Research, King Saud University, Riyadh, Kingdom of Saudi Arabia \\ Correspondence to: Malak Abedalthagafi, email: malthagafi@kfmc.med.sa \\ Keywords: breast cancer; PIK3CA; BCa; Saudi Arabia; BRCA \\ Received: November 17, $2020 \quad$ Accepted: February 19, $2021 \quad$ Published: March 30, 2021
}

Copyright: (c) 2021 Barakeh et al. This is an open access article distributed under the terms of the Creative Commons Attribution License (CC BY 3.0), which permits unrestricted use, distribution, and reproduction in any medium, provided the original author and source are credited.

\section{ABSTRACT}

Breast cancer ( $\mathrm{BCa}$ ) ranks first in incidence rate among cancers in Arab females. The association between genetic polymorphisms in tumor suppressor genes and the risk of BCa has been studied in many ethnic populations with conflicting conclusions while Arab females and Saudi Arabian studies are still lacking. We screened a cohort of Saudi BCa patients by NGS using a bespoke gene panel to clarify the genetic landscape of this population, correlating and assessing genetic findings with clinical outcomes. We identified a total of 263 mutations spanning $\mathbf{5 1}$ genes, including several frequently mutated. Among the genes analyzed, the highest mutation rates were found in PIK3CA (12.9\%), BRCA2 (11.7\%), BRCA1 (10.2\%), TP53 (6.0\%), MSH2 (3.8\%), PMS2 (3.8\%), BARD1 (3.8\%), MLH1 (3.4\%), CDH1 (3.0\%), RAD50 (3.0\%), MSH6 (3.0\%), NF1 (2.6\%), in addition to others. We identified multiple common recurrent variants and previously reported mutations. We also identified 46 novel variants in $\mathbf{2 2}$ genes that were predicted to have a pathogenic effect. Survival analysis according to the four most common mutations (BRCA1, BRCA2, TP53, and PIK3CA) showed reduced survival in BRCA1 and BRCA2-mutant patients compared to total patients. Moreover, BRCA2 was demonstrated as an independent predictor of reduced survival using independent Cox proportional hazard models.

We reveal the landscape of the mutations associated with BCa in Saudi women, highlighting the importance of routine genetic sequencing in implementation of precision therapies in KSA.

\section{INTRODUCTION}

Worldwide, breast cancer is the most common cancer in women. Arab and Middle-Eastern women have a high risk of cancer with an average age at diagnosis of 48 years, which is almost ten years earlier than in western countries
[1]. According to estimates from the GLOBOCAN 2018 database, $\mathrm{BCa}$ ranks second in terms of cancer incidence and is the fourth leading cause of cancer-related mortality worldwide. Approximately 2.1 million new $\mathrm{BCa}$ diagnoses were reported globally by GLOBOCAN in 2018, comprising $11.6 \%$ of all new cancer diagnoses [2]. 
The heterogeneous nature of $\mathrm{BCa}$ has led to the development of a classification system based on expression profiles. Genome-wide RNA expression profiling subdivides $\mathrm{BCa}$ into five classes according to gene expression profiles. The classes are based on the expression of immunohistochemical (IHC) tissue markers as indicated by: (1) estrogen receptor (ER) positivity; (2) progesterone receptor (PR) positivity; (3) human epidermal growth factor receptor 2 (HER2) positivity; (4) proliferation index (marked by the Ki67 protein); and (5) the expression of a unique cluster of genes termed the basal cluster (TNBC) $[3,4]$. The expression patterns of these genes define the molecular signature for each subtype. Accordingly, positive ER and/or PR, negative HER2, and low levels of Ki67 suggest the luminal A BCa subtype, which is the most common and displays the best prognosis. A positive ER and/or PR, either positive HER2 or negative HER2, and high levels of Ki67 suggest a luminal B BCa subtype, which makes up less than $20 \%$ of all $\mathrm{BCa}$ cases and has lower survival rates than luminal A. The absence of ER and PR expression accompanied by high expression of HER2 and proliferation gene clusters and low expression of luminal and basal clusters, as detected by IHC, suggests a HER2-enriched BCa subtype, which accounts for $10 \%$ to $15 \%$ of all cases and has a poorer prognosis than luminal cancers. Negative ER, negative $\mathrm{PR}$, and negative HER2 suggest a triple-negative/ basal-like BCa (TNBC) subtype, which makes up 20\% of all BCas. This subtype is aggressive and manifests at earlier ages [5-9].

Approximately $10-20 \%$ of $\mathrm{BCa}$ patients have at least one affected first-degree relative. Up to $20 \%$ carry germline mutations in the $\mathrm{BCa}$ susceptibility tumor suppressor genes 1 or 2 (BRCA1 or BRCA2). The majority of these mutations are frameshifts that generate premature stop codons and decrease the production of a functional BRCA protein [1]. BRCAs are tumor suppressors that play an important role in DNA damage repair through homology-directed repair (HDR). Mutations in genes other than BRCA tumor suppressors account for less than $1 \%$ of all inherited BCas [10-13]. For instance, ATM mutations are responsible for the development of ataxia telangiectasia (AT). AT patients have a significant potential to develop BCa by the age of 50. The ATM gene is involved in DNA damage repair [14]. Similarly, PALB2 (an interacting partner of BRCA1 and BRCA2 and CHEK2) is known to carry loss-of-function mutations implicated in hereditary $\mathrm{BCa}[15]$.

Numerous signaling pathways involved in healthy development have been implicated in $\mathrm{BCa}$ progression. These pathways are often linked to cell proliferation, apoptosis, differentiation, and motility [16]. Three significant pathways govern mammary gland and $\mathrm{BCa}$ stem cell development: (1) estrogen receptor (ER) signaling; (2) HER2 signaling; and (3) canonical Wnt signaling. In ER signaling, estrogen binds membrane estrogen receptors and triggers a cascade of events that ultimately promote the binding of nuclear estrogen receptors $(E R \alpha, E R \beta)$ with estrogen response elements (EREs). BRCA1 acts as a corepressor and inhibits ER $\alpha$ signaling [17], while cyclin D1 binds to ER $\alpha$ and supports $\mathrm{BCa}$ growth [3]. In HER2 signaling, human epidermal growth factor receptor-2 (HER-2) dimerizes as a result of ligand binding. This leads to the phosphorylation of tyrosine residues in the intracellular domain of HER2 and the activation of downstream pathways, including the mitogen-activated protein kinase (MAPK) and phosphatidylinositol 4,5-bisphosphate 3-kinase (PI3K) pathways [18]. Similarly, binding of the Wnt-receptor to its ligand activates canonical Wnt/ $\beta$-catenin, which subsequently leads to the regulation of oncogenic gene expression, including MYC, CCND1, MMP7 and CD44 [19]. Other pathways involved in $\mathrm{BCa}$ development include cyclin-dependent kinase (CDK) signaling, notch signaling, sonic hedgehog $(\mathrm{SHH})$ signaling, breast tumor kinase (BRK) signaling, and PI3K/AKT/mTOR signaling [20-23].

Many genes that are susceptible to oncogenic mutations are linked to $\mathrm{BCa}$ development. Somatic mutations in PIK3CA account for approximately 30\% of the processes that enhance $\mathrm{PI} 3 \mathrm{~K} / \mathrm{AKT} / \mathrm{mTOR}$ signaling, the most common oncogenic signaling pathway linked to $\mathrm{BCa}$ [24]. In general, PIK3CA mutations are useful prognostic markers and are prevalent in ER-positive/ HER2-negative tumors; there is also new evidence of PIK3CA mutation prevalence in HER2-positive tumors $[25,26]$. The majority of PIK3CA somatic mutations cluster at two hot spots, one in exon 9 (E542K or E545K) and the other in exon 20 (H1047R or H1047L) [27]. Mutations in PIK3R1 are also implicated in BCa, albeit with lower frequencies. Other PI3K-enhancing mechanisms, such as the amplification of HER2, the loss of PTEN function, and the introduction of AKT1 activating mutations, have also been reported [28]. Somatic mutations in TP53 are also frequent in a large number of human BCas [29].

A genomic model for $\mathrm{BCa}$ was generated using nextgeneration sequencing (NGS). Genome-wide association studies (GWAS) have identified various BCa-associated loci. Five risk loci have been reported since 2007 using GWAS, with approximately 1000 loci still unidentified [30]. Two other loci were found to be associated with $\mathrm{BCa}$ in African women in 2013, [31] and three were found to be associated with BCa in Asian women in 2014 [32]. Novel mutations in BRCA1, BRCA2, and PALB2 were also identified in breast and ovarian cancer using whole genome amplification (WGA) [33]. Whole-exome sequencing (WES) was used to detect rare deletions in BRCA2 linked to male BCa risk, [34] and rare mutations in FANCC and BLM were identified as susceptibility alleles for $\mathrm{BCa}$ [35]. WES also facilitated both the identification of the FANCM gene as a susceptible gene 
for triple-negative BCa [36] and the association of XCR1, DLL1, TH, ACCS, SPPL3, CCNF and SRL with BCa. Unlike GWAS and WES, targeted sequencing addresses known loci, allowing accelerated mutation detection rates and accurately targeted therapy [37]. Targeted therapies for $\mathrm{BCa}$ are used to treat patients who overexpress ER, HER1, HER2, and vascular endothelial growth factor (VEGF). Directed therapy includes inhibitors of PI3K/AKT/mTOR, RAS/MEK/ERK, SRC kinase, insulin-like-growth-factor [IGF/IGF-receptor (IGFR)], poly-ADP ribose polymerase (PARP), and matrix metalloproteases (MMPs) [38].

According to the 2014 Saudi Cancer Registry (SCR), BCa is the most prevalent cancer in Saudi women (approximately $28.7 \%$ of all cancers). Approximately $78 \%$ of Saudi BCas are the IDC type. Although Saudi Arabia has a lower age-standardized rate (ASR) for female BCa than Western countries, a stable increase in the incidence of $\mathrm{BCa}$ has been observed, specifically in the Eastern Province [39]. At the molecular level, the most common BCa subtypes in the Saudi population include luminal A (58.5\%), triplenegative (14.8\%), luminal B (14.5\%), and HER2-positive $(12.3 \%)$ [40]. Clinically, these figures represent a robust diagnostic measure that can direct personalized therapy. In this study, we screened a cohort of Saudi BCa patients using a cancer-specific gene panel to ascertain the mutation spectrum and explore the possible clinical implications of the identified somatic variants in $\mathrm{BCa}$ development.

\section{RESULTS}

Fifty-three cases were sequenced. Of the cases, 20 samples (37.7\%) were luminal, 13 samples $(24.5 \%)$ were TNBC, 7 samples (13.2\%) were HER2-enriched and 13 samples $(24.5 \%)$ were not classified (Table 1). Fifty-one samples $(96 \%)$ were IDC, one was diagnosed as IDC with atypical medullary features and one as IDC with micropapillary features. Four samples had a metastatic disease corresponding to stage IV (7.5\%), while remaining samples $(92 \%)$ presented with a localized disease (Supplementary Table 1). Charts analysis revealed that all excision samples have received neoadjuvant therapy (49 patients, 92\%). Adjuvant chemotherapy administered was 3 cycles of FEC100 (5 fluorouracil, epirubicin and cyclophosphamide) and Docetaxa, while patients with luminal tumors received a regimen of Tamoxifen. Adjuvant Radiotherapy was also administered.

\section{Mutations}

Because the validation establishes the reproducible limit of detection at $10 \%$ allele fraction at $50 \times$ coverage, our laboratory has set a minimum tumor content of $20 \%$ neoplastic cell nuclei based on histologic evaluation as a preanalytic criterion for sequencing. Heterozygous somatic variants in a diploid tumor population would be expected to be identified in specimens meeting this criterion. Using an in- house pipeline and tertiary analysis, 263 mutations spanning 51 genes were filtered. The most frequently mutated somatic genes were PIK3CA (12.9\%), BRCA2 (11.7\%), BRCA1 (10.2\%), TP53 (6.0\%), MSH2 (3.8\%), PMS2 (3.8\%), BARD1 (3.8\%), MLH1 (3.4\%), CDH1 (3.0\%), RAD50 (3.0\%), MSH6 (3.0\%), NF1 (2.6\%), RAD51D (2.2\%), ATM (1.5\%), PALB2 (2.6\%), and MLH3 (1.1\%) (Figure 1). The cohort also included common recurrent variants. Recurrent variants included $\mathrm{H} 1047 \mathrm{R}$ in PIK3CA (2.6\% of cases), $\mathrm{N} 550 \mathrm{H}$ in BRCA1 (1.15\% of cases), c.1461 1462delinsCA in BARD1 (70\% of cases), and I2285V in BRCA2 $(0.6 \%$ of cases). On the other hand, $56.6 \%$ of our patients harbored PIK3CA mutations, while BRCA2, BRCA1 and TP53 were mutated in $37.7 \%, 30.18 \%$ and $24.5 \%$, respectively (pathogenic, nonpathogenic and novel) (Table 1).

\section{Known mutations}

We identified 123 previously reported mutations spanning 44 genes in 53 tumor samples. The vast majority of pathogenic variants were found in PIK3CA (24 variants), TP53 (12 variants), BRCA2 (10 variants), and BRCA1 (14 variants). PIK3CA carried the most common recurrent mutation in our sample (p.H1047R). Other pathogenic PIK3CA variants included p.Q546R, p.R412Q, p.E1037K, p.N1044K, p.H1047L, p.M1043I, p.H1065Y, p.E545K, p.R38C, and c.1060-17C >A (Supplementary Table 2).

\section{Novel mutations}

Most of the novel variants identified were in BRCA2 (9 variants), with additional variants in PIK3CA (4 variants), BRCA1 (3 variants), and TP53 (3 variants) (Supplementary Table 2).

\section{Association with clinical characteristics and subtypes}

Associations between common gene mutations (TP53, PIK3CA, BRCA 2 and BRCA1) and clinical characteristics are delineated in Table 2. Mutations in PIK3CA, BRCA1 and BRCA2 showed no significant association with patient age except for TP53 $(p=0.004)$. TP53 mutations were associated with ER- and PR-negative status $(P=0.003)$, in addition to a prominent in situ component. BRCA1 $(P=0.029)$ and BRCA2 $(P=0.038)$ variants were also associated with DCIS. There was no relationship between mutations in PIK3CA, BRCA1 and BRCA2 and subtype. Only the mutation in TP53 was significantly associated with subtype $(p=0.003)$.

\section{Statistical results}

The OS of our study patients was 77\% (5-year overall survival) (Figure 2A). The survival probability was lower 
Table 1: Patient clinical characteristics summary

\begin{tabular}{|c|c|}
\hline Characteristic & Total number \\
\hline Total $(\%)$ & $53(100 \%)$ \\
\hline Age Average (range) & $52.2(32-76)$ \\
\hline Gender & Female \\
\hline Special Histopathology Subtypes & $\begin{array}{l}\text { - IDC with atypical medullary cancer features }(n=1) \text {. } \\
\text { - IDC with micropapillary features }(n=1)\end{array}$ \\
\hline \multicolumn{2}{|l|}{$\mathrm{SBR}^{*}$ GRADE } \\
\hline I & $2(3.7 \%)$ \\
\hline II & $28(52.8 \%)$ \\
\hline III & $23(43.3 \%)$ \\
\hline DCIS & $30(56.6 \%)$ \\
\hline \multicolumn{2}{|l|}{ HORMONE MARKERS } \\
\hline ER/PR (Luminal) & $20(37.7 \%)$ \\
\hline HER2-NEU & $7(13.2 \%)$ \\
\hline TNBC & $13(24.5 \%)$ \\
\hline Unclassified & $13(24.5 \%)$ \\
\hline \multicolumn{2}{|l|}{ COMMON GENES } \\
\hline BRCA1 & $16(30.18 \%)$ \\
\hline BRCA2 & $20(37.7 \%)$ \\
\hline TP53 & $14(26.4 \%)$ \\
\hline PIK3CA & $30(56.6 \%)$ \\
\hline
\end{tabular}

*SBR: Nottingham grading system

for patients with mutant BRCA1 (50\% versus $86 \%$ ); this difference was highly statistically significant $(p=0.004)$ (Figure 2B). Mutation in BRCA2 resulted in a lower survival rate as well $(55.6 \%$ vs $89.9 \% ; p=0.003)$ (Figure $2 \mathrm{C})$. A lower survival rate was observed with mutations in TP53 (64.3\% versus $82.2 \%)$. However, there was no significant difference between the 2 rates (Figure 2D). There was no difference in survival between patients with and without PIK3CA mutations (76.9\% vs 77.3\%) (Figure 2E).

Univariate and multivariate Cox proportional hazards models were used to estimate the hazard ratios of carriers and noncarriers of the mutation (Table 3). Age was included as a prognostic factor in addition to gene mutations. Univariate analysis showed that patients with BRCA1 mutations had a 4.83 -fold increased risk of dying (95\% CI: 1.47-15.91, $P=0.010$ ). Additionally, BRCA2 mutations had a 5.87-fold increased risk compared to that in noncarriers (95\% CI: $1.55-22.21, P=0.009)$. However, multivariate analysis showed BRCA2 to be the only independent factor that significantly contributed to decreased survival $(\mathrm{HR}=$ $5.14,95 \%$ CI 1.16-22.80, $P=0.031)$.

\section{DISCUSSION}

There is a high prevalence of BCa mortality among Saudi Arabian women, and the burden of $\mathrm{BCa}$ in the Arab world continues to grow $[41,42]$. Despite its importance, our knowledge of the risk factors for $\mathrm{BCa}$ in Saudi and
Arab women, in general, remains sparse [43]. The majority of Arab BCa cases are not caused by inherited genetic factors but are associated with somatic mutations in breast cells that accumulate over time [44]. In this study, to determine somatic mutation frequencies in Saudi Arabian women, we sequenced the protein-coding exons of 70 genes in tumor samples from 53 cases. A heavy mutation burden was detected in all BCa tumors. This is potentially a hallmark of increased genomic instability and may correlate with tumor aggressiveness. BRCA1, BRCA2, PIK3CA, and TP53 were the most frequently mutated genes, in agreement with previous studies [8, $45,46]$. PIK3CA was the most common somatic mutation in our cohort, as in other populations, with an additional prominence of TP53 (Figure 1), which aligns with the prevalence previously described in the region. In contrast, we did not detect high-frequency mutations of PTEN and RB1 as noted in other Arab cohorts [47, 48]. This may indicate distinct gene mutation frequencies in the Saudi BCa population.

The study cohort included common recurrent variants, including $\mathrm{N} 550 \mathrm{H}$ in BRCA1 and $\mathrm{H} 1047 \mathrm{R}$ in PIK3CA and P41L in BRCA2 [49-51]. Of the known pathogenic mutations, the majority were found in PIK3CA (24 variants), TP53 (12 variants), BRCA2 (10 variants), and BRCA1 (14 variants).

Genetic testing in $\mathrm{BCa}$ is rapidly advancing, and the ability to identify germline mutations in high-risk 
individuals permits increased surveillance and early genetic counseling. BRCA1 and BRCA2 germline mutations account for approximately $30 \%$ of heritable BCas globally and approximately $20 \%$ of Arab BCas [52-54]. In high-risk Saudi patients, BRCA1/BRCA2 mutation rates of $12.9 \%$ were reported in $\mathrm{BCa}$ tumors [55], while our cohort, on the other hand, had higher somatic mutations rates $(30.18 \%$ for BRCA1 and $37.7 \%$ for BRCA2). Additionally, we report that patients with BRCA1 and BRCA2 mutations had shorter overall survival than patients without these mutations $(p=0.004$ and $p=0.003$, respectively) (Figure $2 \mathrm{~A}$ and $2 \mathrm{~B}$ ). Cox hazard ratio analysis also showed a 4.83- and 5.87-fold increase in hazard ratio in samples with these mutations, while BRCA2 appeared to be the only independent factor contributing significantly to lower survival (Table 3 ).

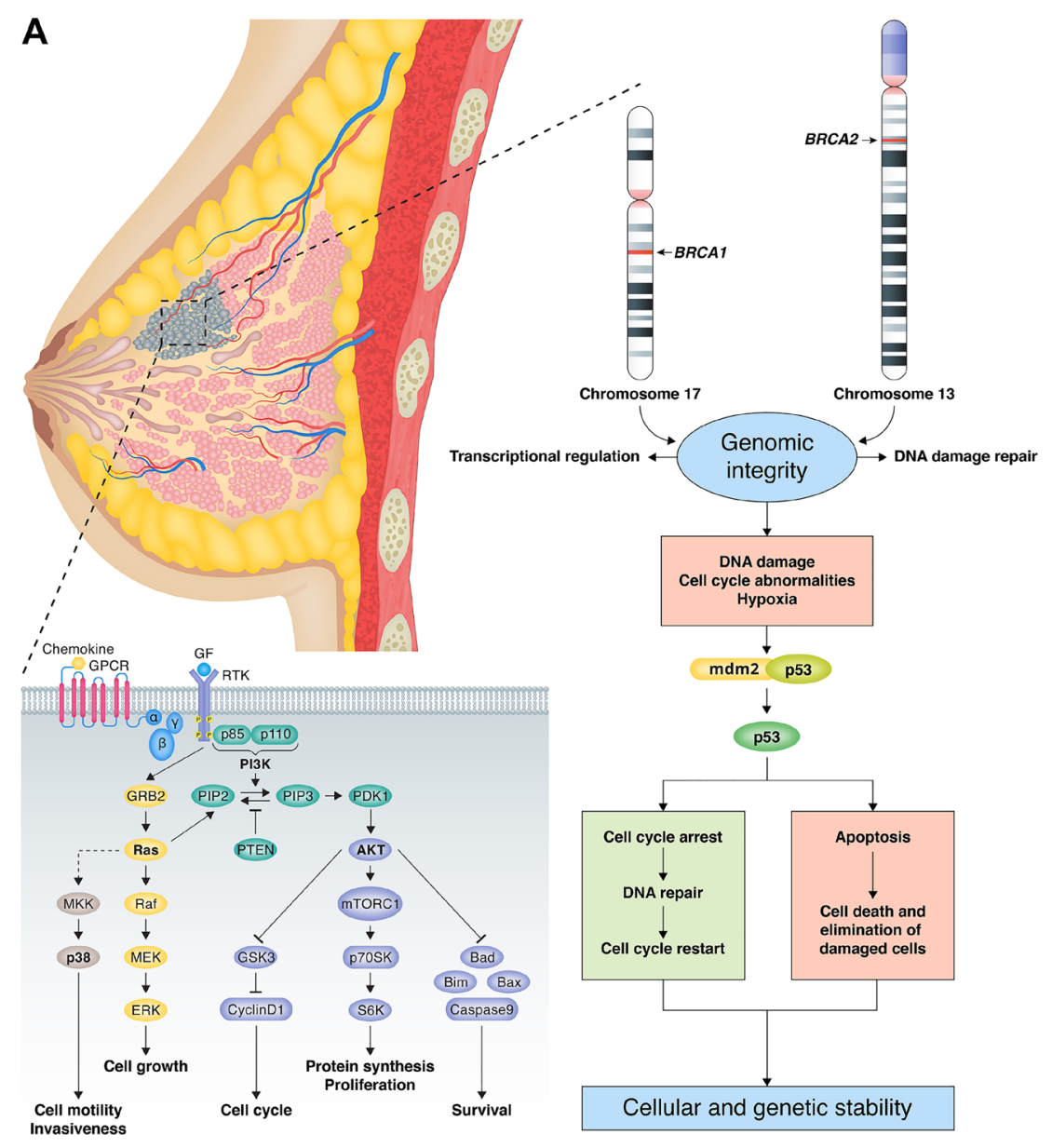

\section{B}

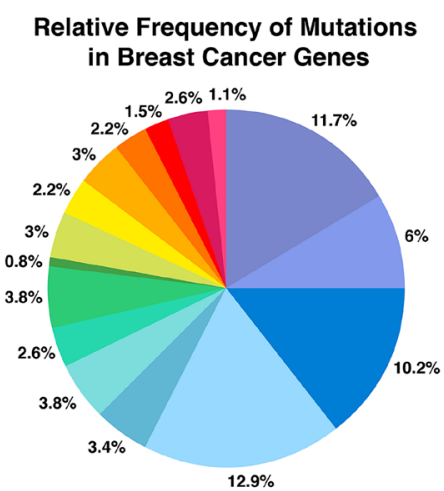

Total Number of Pathogenic Variants

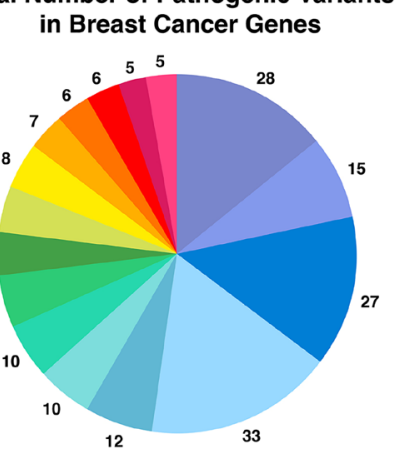

口 BRCA2

TP53

BRCA1

PIK3CA

MLH1

$\square$ MSH2

NF1

- PMS2

APC

CDH1

RAD50

MSH6

RAD51D

- ATM

PALB2

MLH3

Figure 1: (A) BRCA1, BRCA2 and TP53 in DNA damage repair pathway resulting in cellular and genetic instability with potential points for targeted therapy. PIK3CA cellular pathway effects on cell cycle, invasiveness and survival with potential points for targeted therapy. (B) Number and percent of mutations for genes of interest. The most frequently mutated somatic genes were PIK3CA (12.9\%), BRCA2 (11.7\%), BRCA1 (10.2\%), TP53 (6.0\%), MSH2 (3.8\%), PMS2 (3.8\%), BARD1 (3.8\%), MLH1 (3.4\%), CDH1 (3.0\%), RAD50 (3.0\%), MSH6 (3.0\%), NF1 (2.6\%), RAD51D (2.2\%), ATM (1.5\%), PALB2 (2.6\%), and MLH3 (1.1\%). 
Table 2: The association of gene mutations with age, subtype and DCIS

\begin{tabular}{|c|c|c|c|c|c|c|c|c|c|c|c|c|}
\hline & \multicolumn{2}{|c|}{ BRCA1 } & \multicolumn{4}{|c|}{ BRCA2 } & \multicolumn{2}{|c|}{ PIK3CA } & \multicolumn{4}{|c|}{ TP53 } \\
\hline & Mutant & WT & $P$ & Mutant & WT & $P$ & Mutant & WT & $P$ & Mutant & WT & $P$ \\
\hline & $n=15$ & $n=25$ & & $n=17$ & $n=23$ & & $n=22$ & $n=18$ & & $n=9$ & $n=31$ & \\
\hline $\begin{array}{l}\text { HER2 } \\
\text { enriched }\end{array}$ & $3(20 \%)$ & $4(16 \%)$ & & $3(17.65 \%)$ & $4(17.39 \%)$ & & $4(18.18 \%)$ & $3(16.66 \%)$ & & $1(11.11 \%)$ & $6(19.35 \%)$ & \\
\hline Subtype $^{\dagger}$ & & & 0.618 & & & 0.057 & & & 0.838 & & & 0.003 \\
\hline Luminal & $6(40 \%)$ & $14(56 \%)$ & & $5(29.41 \%)$ & $15(65.22 \%)$ & & $12(54.54 \%)$ & $8(44.44 \%)$ & & $1(11.11 \%)$ & $19(61.29 \%)$ & \\
\hline TNBC & $6(40 \%)$ & $7(28 \%)$ & & $9(52.94 \%)$ & $4(17.39 \%)$ & & $6(27.27 \%)$ & $7(38.88 \%)$ & & $7(77.78 \%)$ & $6(19.35 \%)$ & \\
\hline & $n=16$ & $n=36$ & & $n=19$ & $n=33$ & & $n=29$ & $n=23$ & & $n=14$ & $n=38$ & \\
\hline DCIS & & & 0.029 & & & 0.038 & & & 0.232 & & & 0.197 \\
\hline Absent & $9(56.25 \%)$ & $9(25 \%)$ & & $10(52.63 \%)$ & $8(24.24 \%)$ & & $8(27.59 \%)$ & $10(43.48 \%)$ & & $7(50 \%)$ & $11(28.95 \%)$ & \\
\hline Present & $7(43.75 \%)$ & $27(75 \%)$ & & $9(47.37 \%)$ & $25(75.76 \%)$ & & $21(72.41 \%)$ & $13(56.52 \%)$ & & $7(50 \%)$ & $27(71.05 \%)$ & \\
\hline Age & & & 0.132 & & & 0.693 & & & 0.686 & & & 0.004 \\
\hline$<50$ & $4(25 \%)$ & $17(47.22 \%)$ & & $7(36.84 \%)$ & $14(42.42 \%)$ & & $11(37.93 \%)$ & $10(43.48 \%)$ & & $1(7.14 \%)$ & $20(52.63 \%)$ & \\
\hline$\geq 50$ & $12(75 \%)$ & $19(52.78 \%)$ & & $12(63.16 \%)$ & $19(57.58 \%)$ & & $18(62.07 \%)$ & $13(56.52 \%)$ & & $13(92.86 \%)$ & $18(47.37 \%)$ & \\
\hline
\end{tabular}

$\dagger$ Subtype data is available for 40 patients only. WT: Wild Type.

BRCA1 and BRCA2 BCa have therapeutic relevance. As an example, PARP inhibitors are more effective in metastatic $\mathrm{BCa}$ patients with $\mathrm{BRCA} 1 / \mathrm{BRCA} 2$ mutations than in those without these mutations. Olaparib (a PARP inhibitor) is now FDA-approved for treating metastatic $\mathrm{BCa}$ positive for BRCA1/BRCA2 mutations [56]. Further clinical trials investigating the use of cisplatin and olaparib as systemic therapies for BRCA-associated BCas are also underway and are showing favorable results [57-59]. The combination of these therapies with knowledge of lifestyle factors and other personal characteristics may further personalize treatments for BRCA1/BRCA2 BCas, revolutionizing treatment efficacy in the future.

PIK3CA carried the most common mutation across the samples (p.H1047R). Other potential pathogenic PIK3CA variants included p.Q546R, p.R412Q, p.E1037K, p.N1044K, p.H1047L, p.E545K, p.R38C, and c.1060$17 \mathrm{C}>$ A. Gain-of-function mutations in PIK3CA have been identified in many cancers with a global incidence of $26 \%$ and an incidence of approximately $29 \%$ in Arab $\mathrm{BCas}$ [48]. PIK3CA mutations are significantly associated with a lower $\mathrm{BCa}$ grade and hormone receptor positivity in Arab countries [48]. Our cohort did not show a significant difference with regard to these features or survival outcome. In comparison, a recent pooled analysis of $\geq 10,000$ earlystage $\mathrm{BCa}$ patients with PIK3CA-mutated tumors showed an improved prognosis specific to ER, PR+/HER2- and TNBC subtypes, but not the HER2+ subtypes, which were associated with a reduced overall survival [1]. Our cohort included only four HER2+ subtypes, while the remainder of the subtypes included twelve luminal and six triple-negative BCas (Table 2), which reflects a similar behavior in those tumors. Additionally, PIK3CA was found in DCIS in $72.4 \%$ (Table 2), which affirms the reported literature of PIK3CA involvement in early breast carcinogenesis [45, 60]. From a treatment standpoint, the OncoKB and $\mathrm{CIViC}$ websites identified two actionable mutations in PIK3CA. The first was c.3140A $>$ G, p.H1047R or p.H1047L, the most common pathogenic mutation which was altered in nine patients. According to CIViC, this mutation has an actionability score of 49 and should respond to alpelisib and fulvestrant, buparlisib and fulvestrant, fulvestrant and taselisib, alpelisib, buparlisib, copanlisib, GDC-0077, serabelisib and taselisib (OncoKB). The other mutation was c.1633G>A, p.E545K, which has an actionability score of 34 . Genetic testing for PI3KCA mutations may, therefore, aid individualization of BCa treatment in Saudi women [61-63].

According to the literature, TP53 is mutated in approximately $80 \%$ of TNBC tumors [60]. Our cohort reported a $77.7 \%$ frequency of mutations in TNBC cancers $(p=0.003)$, which correlates with previously reported literature. Additionally, TP53 is reported to be associated with poor prognosis in triple-negative cancers [64]; however, our data did not show any significant difference when comparing the OS of carrier and noncarrier patients. Such patients can benefit from immune checkpoint inhibitors (ICIs), as patients with a mutant TP53 and wildtype PIK3CA demonstrate favorable immunotherapyresponsive signatures [65].

Taken together, we identified somatic mutation variants in Saudi BCa patients; BRCA1, BRCA2, TP53, and PIK3CA were found to be among the most common. In total, we identified 39 novel mutations that were not reported before and were predicted to be pathogenic. Our study has pertinent limitations. Further, our limited sample size, particularly for limited somatic genomics aberrations analyzes, may limit generalizability. However, we highlighted the importance of routine genetic sequencing in the implementation of precision therapies in Saudi Arabia. More regional studies are still needed.

\section{MATERIALS AND METHODS}

Tissue samples were collected from 53 consenting Saudi patients in King Fahad Medical City (KFMC) diagnosed with BCa in the period of 2011-2015. All cases 
Table 3: Univariate and multivariate cox proportional hazards model estimations for carriers of the mutation when compared to non-carriers

\begin{tabular}{lcccccc}
\hline Factor & \multicolumn{3}{c}{ Univariate } & \multicolumn{3}{c}{ Multivariate } \\
\cline { 2 - 7 } & HR & $\mathbf{9 5 \%}$ CI & $\boldsymbol{P}$ & HR & $\mathbf{9 5 \%}$ CI & $\boldsymbol{P}$ \\
\hline BRCA1, mutated vs. wild type & 4.83 & $1.47-15.91$ & 0.010 & 3.26 & $0.65-16.38$ & 0.152 \\
BRCA2, mutated vs. wild type & 5.87 & $1.55-22.21$ & 0.009 & 5.14 & $1.16-22.80$ & 0.031 \\
PIK3CA, mutated vs. wild type & 0.97 & $0.30-3.17$ & 0.958 & 1.58 & $0.42-5.92$ & 0.495 \\
TP53, mutated vs. wild type & 2.41 & $0.73-7.90$ & 0.147 & 0.84 & $0.17-4.23$ & 0.829 \\
Age, $\geq$ 50 vs. $<$ 50 & 1.79 & $0.48-6.77$ & 0.388 & 1.12 & $0.25-5.01$ & 0.881 \\
\hline
\end{tabular}

were followed up retrospectively for a median duration of 5 years. Cases were selected based upon cancer tissue content. ER/PR/HER2 immunohistochemical status was obtained from histopathology reports, all reported and published according to the ASCP/CAP guidelines (updated version of 2018). Samples were collected as FFPE (formalin-fixed, paraffin embedded) tumor blocks. FFPE tumors and hematoxylin-eosin-stained slides were prepared and reviewed by a pathologist (MA) to identify areas of $\geq 20 \%$ tumor for molecular analysis. The tumor-
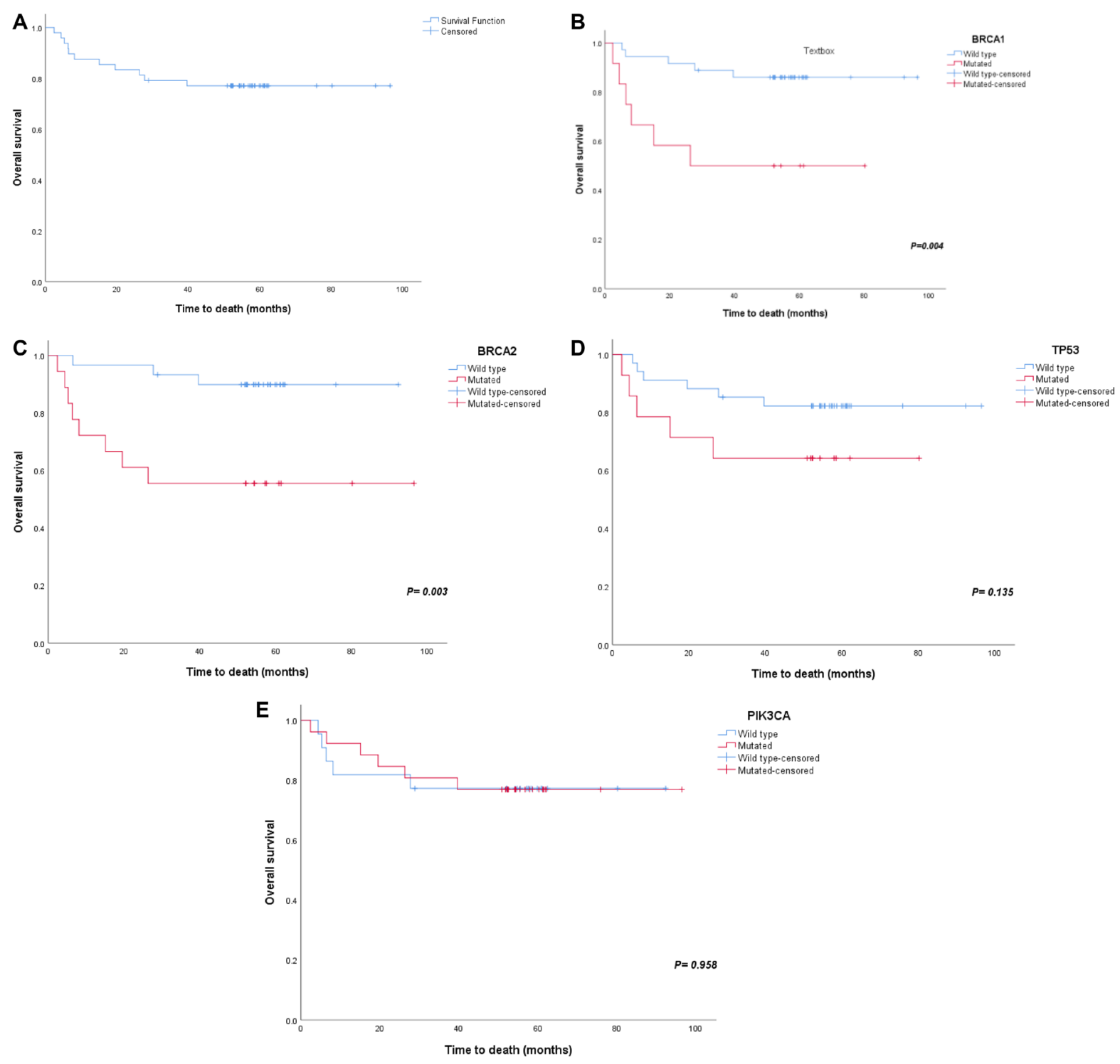

Figure 2: Survival plots for major gene mutations. Major gene mutations have showed effects on survival (A) overall survival, (B) BRCA1 survival, (C) BRCA2 survival, (D) TP53 survival and (E) PIK3CA survival. 
enriched areas were macrodissected or from ten 5-micron tissue sections or punched from the block. In order to obtain sufficient quantities of DNA, several isolations were performed from each sample, pooled to ensure homogeneity, and then aliquoted for use in validation. DNA was manually extracted from the blocks using the GeneRead DNA FFPE Kit (QIAGEN).

All samples underwent targeted sequencing using a customized panel designed by Thermo Fisher Scientific that has been verified for both sensitivity and specificity. The panel includes the following 70 known cancer genes: BRCA2, TP53, BRCA1, PIK3CA, MLH1, ATR, BARD1, ATM, PMS2, RAD50, NF1, MSH2, MSH6, RAD51D, ALK, CDH1, PRF1, RAD51C, APC, MLH3, MYH8, PALB2, PPM1D, PTEN, RET, BRIP1, AXIN2, CDC73, CDK12, CHEK2, EGFR, ELAC2, EXT2, FANCM, GPC3, HOXB13, KIT, PDGFRB, POLE, PTCH1, RB1, RECQL4, TSC1, VHL, AIP, AKT1, BAP1, BMPR1A, BUB1, CBL, DICER1, FANCI, HNF1A, INSRR, LIG4, MEN1, NF2, NTRK1, PALLD, PTCH2, RHBDF2, SETBP1, SMAD4, SMARCB1, SMARCE1, SPRED1, TMEM127, TSC2, WRN and WT1.

The panel was selected for its high coverage of genes related to $\mathrm{BCa}[66]$. The samples were sequenced on the Ion GeneStudio S5 system. Data were generated from the Ion GeneStudio S5 system and underwent initial alignment and analysis by the SHGP pipelines. Pooled sample reads were deconvoluted and sorted using the Picard tools. Reads were aligned to the reference sequence b37 edition from the Human Genome Reference Consortium. Duplicate reads were identified and removed using Picard. Mutation analysis for single-nucleotide variants was performed using MuTect v. 10.2720 and annotated by Oncotator, developed by the Cancer Biology Group at the Broad Institute. For each sequencing run, nonneoplastic FFPE samples were included as controls. Variants identified in these control samples due to sequencing artifacts were filtered.

An average of 1,200 targeted sequencing variants was detected. Variants underwent extensive analysis to identify the single-nucleotide variants (SNVs) of interest. Variants were filtered to exclude those that occur at a populational frequency of greater than $0.1 \%$ in the Exome Sequencing Project database (http://evs.gs.washington. edu/EVS/). The VCF files generated by the system were used for gene and SNV analysis using a specific filtering pipeline as described before [66].

Specific focus was given to genes commonly implicated in $\mathrm{BCa}$, including BRCA1, BRCA2, PIK3CA, TP53, and mismatch repair genes (MMR). Variants were classified into: previously reported variants (those in the Human Gene Mutation Database (HGMD) and/or the Catalogue of Somatic Mutations in Cancer (COSMIC)); novel variants, which are potential pathogenic variants that are not reported in any databases; and polymorphisms: variants not in HGMD or
COSMIC or previously reported in the single nucleotide polymorphism database (dbSNP). Any filtered variants that were reported in COSMIC more than twice were rescued and presented for manual review.

We combined all SNVs identified in the selected genes and listed the genotype for each sample, removed duplicates (recurrent calls), and generated a list including the SNVs identified and the wild-type gene before assessing the association of these changes with selected clinical and pathological characteristics. A sample was considered wild type for a given gene if no mutations were found. Alterations have been previously assessed by mutation-specific PCR, pyrosequencing, or Sanger sequencing.

\section{Statistical analysis}

Chi-square and Fisher's exact tests were performed to examine the relationship between gene mutation and other variables, including age (above or below 50 years), BCa subtype designation (luminal, Her2 enriched, TNBC and unclassified) and DCIS (absent or present). Overall survival (OS) was analyzed by the Kaplan-Meier method; $p$-values were reported using the log-rank test. All cases selected for survival analysis were excisions, and hence, all have received the designated treatments and stages delineated. A Cox proportional hazards regression model was used to calculate the hazard ratios (HRs) and their 95\% CIs for both univariate and multivariate models. All statistical analyses were performed using SPSS 25.0 software (SPSS Inc., Chicago, IL, USA); a $p$-value $<0.05$ was considered statistically significant.

\section{Ethical approval and consent to participate}

We declare that informed consent was obtained from all participants in adherence with the Declaration of Helsinki and the KFMC IRB and Research Advisory Committees (RAC) rules and regulations under the following approved project (KFMC IRB 16-310 MA). All protocols are carried out in accordance with relevant guidelines and regulations. All the methods are approved by the institutional and licensed Ethical Committee of KFMC IRB committee.

\section{Data availability}

The data supporting the results of this article are included within the article and its additional files. Additional datasets used or analyzed during the current study are available from the corresponding author on request.

\section{Abbreviations}

BCa: Breast Cancer; GLOBOCAN: Global Cancer Incidence, Mortality and Prevalence No; IHC: 
Immunohistochemistry; RNA: Ribonucleic Acid; DNA: Deoxyribonucleic Acid; Ki-67: Marker of Proliferation; ER: Estrogen Receptor; PR: Progesterone Receptor; HER2: Human epidermal growth factor receptor 2; HDR: Homology-directed repair; Wnt: Wingless/ Integrated; AT: Ataxia Telangiectasia; ATM: Ataxia Telangiectasia Mutated; ERE: Estrogen response elements; MAPK: Mitogen-activated protein kinase; PI3K: Phosphatidylinositol 4,5-bisphosphate 3-kinase; SHH: Sonic hedgehog; BRK: Breast tumor kinase; NGS: Next-generation sequencing; GWAS: Genomewide association studies; WGA: Whole genome amplification; WES: Whole-exome sequencing; VEGF: Vascular endothelial growth factor; IGF: Insulin-likegrowth-factor; PARP: Poly-ADP ribose polymerase; MMP: Matrix metalloproteases; SCR: Saudi Cancer Registry; ASR: Age-standardized rate; KFMC: King Fahad Medical City; ASCP: American society for clinical pathology; CAP: College of American Pathologists; FFPE: Formalin-fixed, paraffin embedded; SNVs: Single-nucleotide variants; VCF: Virtual Contact File; MMR: Mismatch repair genes; HGMD: Human Gene Mutation Database; COSMIC: Catalogue of Somatic Mutations in Cancer; dbSNP: Single nucleotide polymorphism database; PCR: Polymerase Chain Reaction; TNBC: Triple Negative Breast Cancer; DCIS: Ductal Carcinoma In Situ; HR: Hazard Ratios; OS: Overall survival; IDC: Invasive ductal carcinoma; BRCA1/2: Breast Cancer Gene 1/2; TP53: Tumor Protein 53; FDA: Food and drug administration.

\section{Author contributions}

DB collected and analyzed the clinical data and wrote part of the manuscript. LM, RA, AA, and FA performed the NGS experiments and analyzed the data. YB performed statistical analysis and wrote part of the manuscript. RA, AA and FA performed the NGS experiments and analyzed the data. MA designed the study, analyzed the data, and wrote the manuscript. MA, AM, MR and FA helped with clinical data collection and wrote part of the manuscript. All authors approved the final manuscript.

\section{ACKNOWLEDGMENTS}

All NGS library building, sequencing, and bioinformatics analyses were performed by the Saudi Human Genome Program (SHGP) at the Genomics Research Department at King Fahad Medical City (KFMC). The study was financially supported by King Saud University, Vice Deanship of Research Chairs. We thank Dr. Nada Altassan and the SHGP team in the KFSHRC genetics department for their technical and scientific support.

\section{CONFLICTS OF INTEREST}

The authors have no competing interests or other interests that might be perceived to influence the results and discussion reported in this paper.

\section{FUNDING}

This work was funded and supported by KFMC and (17-65 MA) and the Saudi Human Genome Program at KACST (MA). The funding body was not involved in any stage of the study and had no role in the design of the study, the collection, analysis, and interpretation of data or the writing of the manuscript.

\section{REFERENCES}

1. Rebbeck TR, Friebel TM, Friedman E, Hamann U, Huo D, Kwong A, Olah E, Olopade OI, Solano AR, Teo SH, Thomassen M, Weitzel JN, Chan TL, et al, and EMBRACE, and GEMO Study Collaborators, and HEBON. Mutational spectrum in a worldwide study of 29,700 families with BRCA1 or BRCA2 mutations. Hum Mutat. 2018; 39:593620. https://doi.org/10.1002/humu.23406. [PubMed]

2. Bray F, Ferlay J, Soerjomataram I, Siegel RL, Torre LA, Jemal A. Global cancer statistics 2018: GLOBOCAN estimates of incidence and mortality worldwide for 36 cancers in 185 countries. CA Cancer J Clin. 2018; 68:394424. https://doi.org/10.3322/caac.21492. [PubMed]

3. Feng Y, Spezia M, Huang S, Yuan C, Zeng Z, Zhang L, Ji X, Liu W, Huang B, Luo W, Liu B, Lei Y, Du S, et al. Breast cancer development and progression: Risk factors, cancer stem cells, signaling pathways, genomics, and molecular pathogenesis. Genes Dis. 2018; 5:77-106. https://doi. org/10.1016/j.gendis.2018.05.001. [PubMed]

4. Bertucci F, Finetti P, Birnbaum D. Basal breast cancer: a complex and deadly molecular subtype. Curr Mol Med. 2012; 12:96-110. https://doi. org/10.2174/156652412798376134. [PubMed]

5. Escala-Garcia M, Guo Q, Dork T, Canisius S, Keeman R, Dennis J, Beesley J, Lecarpentier J, Bolla MK, Wang Q, Abraham J, Andrulis IL, Anton-Culver H, et al, and NBCS Collaborators. Genome-wide association study of germline variants and breast cancer-specific mortality. Br J Cancer. 2019; 120:647-57. https://doi.org/10.1038/s41416-0190393-x. [PubMed]

6. Fallahpour S, Navaneelan T, De P, Borgo A. Breast cancer survival by molecular subtype: a population-based analysis of cancer registry data. CMAJ Open. 2017; 5:E734-E9. https://doi.org/10.9778/cmajo.20170030. [PubMed]

7. Howlader N, Cronin KA, Kurian AW, Andridge R. Differences in Breast Cancer Survival by Molecular Subtypes in the United States. Cancer Epidemiol Biomarkers Prev. 2018; 27:619-26. https://doi. org/10.1158/1055-9965.EPI-17-0627. [PubMed] 
8. Encinas G, Sabelnykova VY, de Lyra EC, Hirata Katayama ML, Maistro S, de Vasconcellos Valle PWM, de Lima Pereira GF, Rodrigues LM, de Menezes Pacheco Serio PA, de Gouvea A, Geyer FC, Basso RA, Pasini FS, et al. Somatic mutations in early onset luminal breast cancer. Oncotarget. 2018; 9:22460-79. https://doi.org/10.18632/ oncotarget.25123. [PubMed]

9. Liu Z, Li M, Jiang Z, Wang X. A Comprehensive Immunologic Portrait of Triple-Negative Breast Cancer. Transl Oncol. 2018; 11:311-29. https://doi.org/10.1016/j. tranon.2018.01.011. [PubMed]

10. Easton D. Breast cancer genes--what are the real risks? Nat Genet. 1997; 16:210-1. https://doi.org/10.1038/ng0797210. [PubMed]

11. de Jong MM, Nolte IM, te Meerman GJ, van der Graaf WT, Oosterwijk JC, Kleibeuker JH, Schaapveld M, de Vries EG. Genes other than BRCA1 and BRCA2 involved in breast cancer susceptibility. J Med Genet. 2002; 39:225-42. https://doi.org/10.1136/jmg.39.4.225. [PubMed]

12. Nusbaum R, Vogel KJ, Ready K. Susceptibility to breast cancer: hereditary syndromes and low penetrance genes. Breast Dis. 2007; 27:21-50. https://doi.org/10.3233/bd2007-27103. [PubMed]

13. Hoskins KF, Stopfer JE, Calzone KA, Merajver SD, Rebbeck TR, Garber JE, Weber BL. Assessment and counseling for women with a family history of breast cancer. A guide for clinicians. JAMA. 1995; 273:577-85. [PubMed]

14. Easton DF. The inherited component of cancer. $\mathrm{Br}$ Med Bull. 1994; 50:527-35. https://doi.org/10.1093/ oxfordjournals.bmb.a072908. [PubMed]

15. AlHarthi FS, Qari A, Edress A, Abedalthagafi $M$. Familial/inherited cancer syndrome: a focus on the highly consanguineous Arab population. NPJ Genom Med. 2020; 5:3. https://doi.org/10.1038/s41525-019-0110-y. [PubMed]

16. Tharmapalan P, Mahendralingam M, Berman HK, Khokha R. Mammary stem cells and progenitors: targeting the roots of breast cancer for prevention. EMBO J. 2019; 38:e100852. https://doi.org/10.15252/embj.2018100852. [PubMed]

17. Fan S, Ma YX, Wang C, Yuan RQ, Meng Q, Wang JA, Erdos M, Goldberg ID, Webb P, Kushner PJ, Pestell RG, Rosen EM. Role of direct interaction in BRCA1 inhibition of estrogen receptor activity. Oncogene. 2001; 20:77-87. https://doi.org/10.1038/sj.onc.1204073. [PubMed]

18. Burgess AW. EGFR family: structure physiology signalling and therapeutic targets. Growth Factors. 2008; 26:263-74. https://doi.org/10.1080/08977190802312844. [PubMed]

19. Clevers H, Nusse R. Wnt/beta-catenin signaling and disease. Cell. 2012; 149:1192-205. https://doi.org/10.1016/j. cell.2012.05.012. [PubMed]

20. Lee JJ, Loh K, Yap YS. PI3K/Akt/mTOR inhibitors in breast cancer. Cancer Biol Med. 2015; 12:342-54. https:// doi.org/10.7497/j.issn.2095-3941.2015.0089. [PubMed]
21. Riobo-Del Galdo NA, Lara Montero A, Wertheimer EV. Role of Hedgehog Signaling in Breast Cancer: Pathogenesis and Therapeutics. Cells. 2019; 8:375. https:// doi.org/10.3390/cells8040375. [PubMed]

22. Spring LM, Wander SA, Zangardi M, Bardia A. CDK 4/6 Inhibitors in Breast Cancer: Current Controversies and Future Directions. Curr Oncol Rep. 2019; 21:25. https:// doi.org/10.1007/s11912-019-0769-3. [PubMed]

23. Lukong KE, Richard S. Breast tumor kinase BRK requires kinesin-2 subunit KAP3A in modulation of cell migration. Cell Signal. 2008; 20:432-42. https://doi.org/10.1016/j. cellsig.2007.11.003. [PubMed]

24. Shimoi T, Hamada A, Yamagishi M, Hirai M, Yoshida M, Nishikawa T, Sudo K, Shimomura A, Noguchi E, Yunokawa M, Yonemori K, Shimizu C, Kinoshita T, et al. PIK3CA mutation profiling in patients with breast cancer, using a highly sensitive detection system. Cancer Sci. 2018; 109:2558-66. https://doi.org/10.1111/cas.13696. [PubMed]

25. Berger C, Qian Y, Chen X. The p53-estrogen receptor loop in cancer. Curr Mol Med. 2013; 13:1229-40. https://doi.org /10.2174/15665240113139990065. [PubMed]

26. Elwy F, Helwa R, El Leithy AA, Shehab El din Z, Assem MM, Hassan NH. PIK3CA mutations in HER2-positive Breast Cancer Patients; Frequency and Clinicopathological Perspective in Egyptian Patients. Asian Pac J Cancer Prev. 2017; 18:57-64. https://doi.org/10.22034/ APJCP.2017.18.1.57. [PubMed]

27. Zhang L, Shi L, Zhao X, Wang Y, Yue W. PIK3CA gene mutation associated with poor prognosis of lung adenocarcinoma. Onco Targets Ther. 2013; 6:497-502. https://doi.org/10.2147/OTT.S41643. [ubMed]

28. Mukohara T. PI3K mutations in breast cancer: prognostic and therapeutic implications. Breast Cancer (Dove Med Press). 2015; 7:111-23. https://doi.org/10.2147/bctt.s60696. [PubMed]

29. Zhang Y, Xiong S, Liu B, Pant V, Celii F, Chau G, ElizondoFraire AC, Yang P, You MJ, El-Naggar AK, Navin NE, Lozano G. Somatic Trp53 mutations differentially drive breast cancer and evolution of metastases. Nat Commun. 2018; 9:3953. https://doi.org/10.1038/s41467-018-06146-9. [PubMed]

30. Easton DF, Pooley KA, Dunning AM, Pharoah PD, Thompson D, Ballinger DG, Struewing JP, Morrison J, Field H, Luben R, Wareham N, Ahmed S, Healey CS, et $\mathrm{al}$, and SEARCH collaborators, and kConFab, and AOCS Management Group. Genome-wide association study identifies novel breast cancer susceptibility loci. Nature. 2007; 447:1087-93. https://doi.org/10.1038/nature05887. [PubMed]

31. Chen F, Chen GK, Stram DO, Millikan RC, Ambrosone CB, John EM, Bernstein L, Zheng W, Palmer JR, Hu JJ, Rebbeck TR, Ziegler RG, Nyante S, et al. A genome-wide association study of breast cancer in women of African ancestry. Hum Genet. 2013; 132:39-48. https://doi. org/10.1007/s00439-012-1214-y. [PubMed] 
32. Cai Q, Zhang B, Sung H, Low SK, Kweon SS, Lu W, Shi J, Long J, Wen W, Choi JY, Noh DY, Shen CY, Matsuo K, et al, and DRIVE GAME-ON Consortium. Genome-wide association analysis in East Asians identifies breast cancer susceptibility loci at $1 \mathrm{q} 32.1,5 \mathrm{q} 14.3$ and $15 \mathrm{q} 26.1$. Nat Genet. 2014; 46:886-90. https://doi.org/10.1038/ng.3041. [PubMed]

33. Kraus C, Hoyer J, Vasileiou G, Wunderle M, Lux MP, Fasching PA, Krumbiegel M, Uebe S, Reuter M, Beckmann MW, Reis A. Gene panel sequencing in familial breast/ ovarian cancer patients identifies multiple novel mutations also in genes others than BRCA1/2. Int J Cancer. 2017; 140:95-102. https://doi.org/10.1002/ijc.30428. [PubMed]

34. Timoteo AR, Albuquerque BM, Moura PC, Ramos CC, Agnez-Lima LF, Walsh T, King MC, Lajus TB. Identification of a new BRCA2 large genomic deletion associated with high risk male breast cancer. Hered Cancer Clin Pract. 2015; 13:2. https://doi.org/10.1186/s13053-014-0022-x. [PubMed]

35. Thompson ER, Doyle MA, Ryland GL, Rowley SM, Choong DY, Tothill RW, Thorne H, Barnes DR, Li J, Ellul J, Philip GK, Antill YC, James PA, et al, and kConFab. Exome sequencing identifies rare deleterious mutations in DNA repair genes FANCC and BLM as potential breast cancer susceptibility alleles. PLoS Genet. 2012; 8:e1002894. https://doi.org/10.1371/journal.pgen.1002894. [PubMed]

36. Kiiski JI, Pelttari LM, Khan S, Freysteinsdottir ES, Reynisdottir I, Hart SN, Shimelis H, Vilske S, Kallioniemi A, Schleutker J, Leminen A, Butzow R, Blomqvist C, et al. Exome sequencing identifies FANCM as a susceptibility gene for triple-negative breast cancer. Proc Natl Acad Sci U S A. 2014; 111:15172-7. https://doi.org/10.1073/ pnas.1407909111. [PubMed]

37. Noh JM, Kim J, Cho DY, Choi DH, Park W, Huh SJ. Exome sequencing in a breast cancer family without BRCA mutation. Radiat Oncol J. 2015; 33:149-54. https://doi. org/10.3857/roj.2015.33.2.149. [PubMed]

38. Masoud V, Pagès G. Targeted therapies in breast cancer: New challenges to fight against resistance. World J Clin Oncol. 2017; 8:120-34. https://doi.org/10.5306/wjco. v8.i2.120. [PubMed]

39. AL-Mulhim F, Bakr R, Almedallah D, Alkaltham N, Alotaibi A, Alnoaim S. Screening mammography and breast self-examination: Attitudes and practices of women in the Eastern Province of Saudi Arabia. Saudi J Health Sci. 2018; 7:89-100. https://doi.org/10.4103/sjhs.sjhs_4_18.

40. Alnegheimish NA, Alshatwi RA, Alhefdhi RM, Arafah MM, AlRikabi AC, Husain S. Molecular subtypes of breast carcinoma in Saudi Arabia. A retrospective study. Saudi Med J. 2016; 37:506-12. https://doi.org/10.15537/ smj.2016.5.15000. [PubMed]

41. Hashim MJ, Al-Shamsi FA, Al-Marzooqi NA, Al-Qasemi SS, Mokdad AH, Khan G. Burden of Breast Cancer in the Arab World: Findings from Global Burden of Disease, 2016. J Epidemiol Glob Health. 2018; 8:54-8. https://doi. org/10.2991/j.jegh.2018.09.003. [PubMed]
42. Alotaibi RM, Rezk HR, Juliana CI, Guure C. Breast cancer mortality in Saudi Arabia: Modelling observed and unobserved factors. PLoS One. 2018; 13:e0206148. https:// doi.org/10.1371/journal.pone.0206148. [PubMed]

43. Aghassi-Ippen M, Green MS, Shohat T. Familial risk factors for breast cancer among Arab women in Israel. Eur J Cancer Prev. 2002; 11:327-31. https://doi.org/10.1097/00008469200208000-00003. [PubMed]

44. Weigelt B, Bi R, Kumar R, Blecua P, Mandelker DL, Geyer FC, Pareja F, James PA, Couch FJ, Eccles DM, Blows F, Pharoah P, Li A, Selenica P, et al, and kConFab Investigators. The Landscape of Somatic Genetic Alterations in Breast Cancers From ATM Germline Mutation Carriers. J Natl Cancer Inst. 2018; 110:1030-4. https://doi.org/10.1093/jnci/djy028. [PubMed]

45. Bai X, Zhang E, Ye H, Nandakumar V, Wang Z, Chen L, Tang C, Li J, Li H, Zhang W, Han W, Lou F, Zhang D, et al. PIK3CA and TP53 gene mutations in human breast cancer tumors frequently detected by ion torrent DNA sequencing. PLoS One. 2014; 9:e99306. https://doi.org/10.1371/journal. pone.0099306. [PubMed]

46. Encinas G, Maistro S, Pasini FS, Katayama ML, Brentani MM, Bock GH, Folgueira MA. Somatic mutations in breast and serous ovarian cancer young patients: a systematic review and meta-analysis. Rev Assoc Med Bras (1992). 2015; 61:474-83. https://doi.org/10.1590/18069282.61.05.474. [PubMed]

47. Al-Qasem AJ, Toulimat M, Eldali AM, Tulbah A, Al-Yousef N, Al-Daihan SK, Al-Tassan N, Al-Tweigeri T, Aboussekhra A. TP53 genetic alterations in Arab breast cancer patients: Novel mutations, pattern and distribution. Oncol Lett. 2011; 2:363-9. https://doi.org/10.3892/ol.2011.236. [PubMed]

48. Karakas B, Colak D, Kaya N, Ghebeh H, Al-Qasem A, Hendrayani F, Toulimat M, Al-Tweigeri T, Park BH, Aboussekhra A. Prevalence of PIK3CA mutations and the SNP rs17849079 in Arab breast cancer patients. Cancer Biol Ther. 2013; 14:888-96. https://doi.org/10.4161/cbt.25945. [PubMed]

49. El Saghir NS, Zgheib NK, Assi HA, Khoury KE, Bidet Y, Jaber SM, Charara RN, Farhat RA, Kreidieh FY, Decousus S, Romero P, Nemer GM, Salem Z, et al. BRCA1 and BRCA2 mutations in ethnic Lebanese Arab women with high hereditary risk breast cancer. Oncologist. 2015; 20:357-64. https://doi.org/10.1634/ theoncologist.2014-0364. [PubMed]

50. Evron E, Ben-David AM, Goldberg H, Fried G, Kaufman B, Catane R, Pfeffer MR, Geffen DB, Chernobelsky P, Karni T, Abdah-Bortnyak R, Rosengarten O, Matceyevsky D, et al. Prophylactic irradiation to the contralateral breast for BRCA mutation carriers with early-stage breast cancer. Ann Oncol. 2019; 30:412-7. https://doi.org/10.1093/annonc/ mdy515. [PubMed]

51. Gornstein EL, Sandefur S, Chung JH, Gay LM, Holmes O, Erlich RL, Soman S, Martin LK, Rose AV, Stephens PJ, Ross JS, Miller VA, Ali SM, Blau S. BRCA2 Reversion 
Mutation Associated With Acquired Resistance to Olaparib in Estrogen Receptor-positive Breast Cancer Detected by Genomic Profiling of Tissue and Liquid Biopsy. Clin Breast Cancer. 2018; 18:184-8. https://doi.org/10.1016/j. clbc.2017.12.010. [PubMed]

52. Abbas S, Siddique A, Shahid N, Khan RT, Fatima W. Breast cancer risk associated with BRCA1/2 variants in the Pakistani population. Breast Cancer. 2019; 26:365-72. https://doi.org/10.1007/s12282-018-0932-y. [PubMed]

53. Torres D, Bermejo JL, Rashid MU, Briceno I, Gil F, Beltran A, Ariza V, Hamann U. Prevalence and Penetrance of BRCA1 and BRCA2 Germline Mutations in Colombian Breast Cancer Patients. Sci Rep. 2017; 7:4713. https://doi. org/10.1038/s41598-017-05056-y. [PubMed]

54. Abdulrashid K, AlHussaini N, Ahmed W, Thalib L. Prevalence of BRCA mutations among hereditary breast and/or ovarian cancer patients in Arab countries: systematic review and meta-analysis. BMC Cancer. 2019; 19:256. https://doi.org/10.1186/s12885-019-5463-1. [PubMed]

55. Abulkhair O, Al Balwi M, Makram O, Alsubaie L, Faris M, Shehata H, Hashim A, Arun B, Saadeddin A, Ibrahim E. Prevalence of BRCA1 and BRCA2 Mutations Among High-Risk Saudi Patients With Breast Cancer. J Glob Oncol. 2018; 4:1-9. https://doi.org/10.1200/JGO.18.00066. [PubMed]

56. Das M. Olaparib provides benefit in metastatic breast cancer. Lancet Oncol. 2017; 18:e376. https://doi. org/10.1016/S1470-2045(17)30453-9. [PubMed]

57. Nicolas E, Bertucci F, Sabatier R, Goncalves A. Targeting BRCA Deficiency in Breast Cancer: What are the Clinical Evidences and the Next Perspectives? Cancers (Basel). 2018; 10:E506. https://doi.org/10.3390/cancers 10120506. [PubMed]

58. Tung NM, Garber JE. BRCA1/2 testing: therapeutic implications for breast cancer management. Br J Cancer. 2018; 119:141-52. https://doi.org/10.1038/s41416-0180127-5. [PubMed]

59. Griguolo G, Dieci MV, Guarneri V, Conte P. Olaparib for the treatment of breast cancer. Expert Rev Anticancer Ther. 2018; 18:519-30. https://doi.org/10.1080/14737140.2018.1 458613. [PubMed]
60. Cancer Genome Atlas Network. Comprehensive molecular portraits of human breast tumours. Nature. 2012; 490:6170. https://doi.org/10.1038/nature11412. [PubMed]

61. Fan H, Li C, Xiang Q, Xu L, Zhang Z, Liu Q, Zhang T, Zhou Y, Zhao X, Cui Y. PIK3CA mutations and their response to neoadjuvant treatment in early breast cancer: A systematic review and meta-analysis. Thorac Cancer. 2018; 9:571-9. https://doi.org/10.1111/1759-7714.12618. [PubMed]

62. Desriani, Al-Ahwani F. The sensitivity and efficacy method of PIK3CA exon 9 E545A as a high diagnostic accuracy in breast cancer. J Genet Eng Biotechnol. 2018; 16:71-6. https://doi.org/10.1016/j.jgeb.2017.10.002. [PubMed]

63. Cocciolone V, Cannita K, Tessitore A, Mastroiaco V, Rinaldi L, Paradisi S, Irelli A, Baldi PL, Sidoni T, Ricevuto E, Dal Mas A, Calvisi G, Coletti G, et al. Neoadjuvant chemotherapy in breast cancer: a dose-dense schedule in real life and putative role of PIK3CA mutations. Oncotarget. 2018; 9:27380-96. https://doi.org/10.18632/ oncotarget.25270. [PubMed]

64. Li JP, Zhang XM, Zhang Z, Zheng LH, Jindal S, Liu YJ. Association of p53 expression with poor prognosis in patients with triple-negative breast invasive ductal carcinoma. Medicine (Baltimore). 2019; 98:e15449. https:// doi.org/10.1097/MD.0000000000015449. [PubMed]

65. Cheng J, Ding X, Xu S, Zhu B, Jia Q. Gene expression profiling identified TP53(Mut)PIK3CA(Wild) as a potential biomarker for patients with triple-negative breast cancer treated with immune checkpoint inhibitors. Oncol Lett. 2020; 19:2817-24. https://doi.org/10.3892/ol.2020.11381.

66. AlSahlawi A, Aljelaify R, Magrashi A, AlSaeed M, Almutairi A, Alqubaishi F, Alturkistani A, AlObaid A, Abouelhoda M, AlMubarak L, AlTassan N, Abedalthagafi M. New insights into the genomic landscape of meningiomas identified FGFR3 in a subset of patients with favorable prognoses. Oncotarget. 2019; 10:5549-59. https:// doi.org/10.18632/oncotarget.27178. [PubMed] 